DOI: https://doi.org/10.34069/AI/2021.39.03.9

\title{
Emprendimiento de la tercera edad en Rusia: situación y perspectivas
}

\section{Entrepreneurship for the elderly in Russia: situation and prospects}

Received: February 24, 2021

\author{
Accepted: April 10, 2021
}

\author{
Written by: \\ I.S. Pinkovetskaia ${ }^{32}$ \\ https://orcid.org/0000-0002-8224-9031 \\ Diego Felipe Arbeláez-Campillo ${ }^{33}$ \\ https://orcid.org/0000-0002-9041-9563 \\ Magda Julissa Rojas-Bahamón ${ }^{34}$ \\ https://orcid.org/0000-0003-4882-1476 \\ Elena Sverdlikova ${ }^{35}$
}

\section{Resumen}

El creciente número de personas en edad de jubilación y prejubilación, característico de Rusia, requiere una solución al problema de su empleo, incluso mediante el desarrollo del espíritu empresarial en este grupo de población. El objetivo del estudio fue identificar las características de las actividades de los emprendedores en la tercera edad en Rusia y la dinámica de este emprendimiento en los últimos años y en el futuro. La información estadística sobre la estructura de edad de la población, así como el pronóstico demográfico correspondiente para Rusia, se utilizó como datos iniciales en el estudio. En el transcurso del estudio, se consideraron las características del emprendimiento temprano en la tercera edad, se evaluaron sus niveles para el período de 2013 a 2018. Los resultados del estudio indican una disminución constante en la proporción de emprendedores tempranos de la tercera edad en la población total de Rusia de 2013 a 2018. Se propone una previsión del número potencial de emprendedores de nueva creación para el futuro hasta 2035 según tres opciones. No todas las personas mayores pueden ser emprendedores de la tercera edad, ya que no todos tienen las capacidades e intenciones correspondientes y están preparados para crear sus propios negocios.

Palabras clave: emprendimiento temprano; tercera edad; edad de prejubilación y jubilación; envejecimiento de la población; pronóstico.

\begin{abstract}
The increasing number of people of retirement and pre-retirement age, characteristic of Russia, requires a solution to the problem of their employment, including through the development of entrepreneurship in this population group. The objective of the study was to identify the characteristics of the activities of entrepreneurs in the elderly in Russia and the dynamics of this venture in recent years and in the future. Statistical information on the age structure of the population, as well as the corresponding demographic forecast for Russia, was used as initial data in the study. During the course of the study, the characteristics of early-age entrepreneurship were considered, their levels were evaluated for the period from 2013 to 2018 . The results of the study indicate a constant decrease in the proportion of early-age entrepreneurs in the Total population of Russia from 2013 to 2018. A forecast of the potential number of start-up entrepreneurs for the future up to 2035 is proposed according to three options. Not all seniors can be senior entrepreneurs, as not everyone has the corresponding capabilities and intentions and is ready to create their own businesses.
\end{abstract}

Keywords: early entrepreneurship; third age; early retirement and retirement age; aging of the population; forecast.

\footnotetext{
${ }^{32} \mathrm{PhD}$, Associate Professor, Department of Economic analysis and state management, Ulyanovsk State University, Ulyanovsk, Russia.

${ }^{33}$ Grupo de Investigación Lenguajes, Representaciones y Educación, Universidad de la Amazonia, Colombia.

${ }^{34}$ IE Jorge Eliecer Gaitán, Universidad de la Amazonia, Colombia.

${ }^{35} \mathrm{PhD}$, Associate Professor, Lomonosov Moscow State University, Moscow, Russia.
} 


\section{Introducción}

El envejecimiento de la población se ha convertido en un fenómeno global en los últimos años: según las previsiones desarrolladas en la ONU (World Population Aging, 2019), para 2050 el número de personas mayores se duplicará hasta los 1.500 millones. Al mismo tiempo, casi todos los países del mundo han experimentado un aumento en el número y la proporción de personas mayores en la población. Este cambio dramático en la estructura de edad de la población se debe a una disminución de la mortalidad y está asociado con un aumento en el nivel de salud pública, avances médicos y el desarrollo económico y social de la mayoría de los países. En las economías avanzadas, el hambre, las enfermedades, las lesiones y la muerte prematura, que tienen una esperanza de vida humana limitada a lo largo de la historia de la humanidad, tienen un impacto menor en las personas. La situación se ve agravada por la disminución simultánea de la fecundidad. Es por eso que la ONU ha reconocido el envejecimiento de la población como una mega tendencia demográfica.

La población mundial está envejeciendo y este fenómeno tiene importantes consecuencias para la estructura de la población en edad de trabajar. Para 2035, se espera que el número de personas de 55 años o más supere a todos los niños de 0 a 14 años, y para 2080, a toda la población infantil y juvenil de 0 a 24 años. Como consecuencia directa del envejecimiento de la población, se espera que aumente el número de trabajadores mayores de 55 a 64 años y que represente casi una cuarta parte de la población económicamente activa para 2030 (Harasty y Ostermeier, 2020).

El término tercera edad se utiliza en publicaciones científicas para caracterizar a las personas mayores que pueden participar en la fuerza laboral. Esta edad, según la mayoría de los investigadores, comienza en el rango de 50 a 60 años (Gimmon et al., 2018). Esto implica que para este momento las personas ya han vivido etapas como la infancia, la adolescencia, el estudio (correspondiente a la primera edad), y también han participado en la actividad laboral activa, criando hijos (correspondiente a la segunda edad). Teniendo en cuenta que la duración la tercera edad para los países económicamente desarrollados es de 10 a 30 años. Una característica de esta edad, como parte del camino de la vida de las personas, es su capacidad para satisfacer de forma independiente sus diversos intereses y necesidades, con el objetivo de resolver problemas personales, sociales, psicológicos y económicos. La tendencia al envejecimiento de la población y, en consecuencia, el aumento de la proporción de ciudadanos en edad de prejubilación y jubilación, despiertan un interés considerable por los problemas de las personas de la tercera edad.

La principal dirección para superar el problema del envejecimiento de la población es la extensión de la actividad laboral de las personas (Maestas, Zissimopoulos, 2010). Sin embargo, existen limitaciones a las que se enfrentan las personas mayores al intentar seguir trabajando (Loretto, 2010). La discriminación por edad sigue siendo típica de las economías modernas (Hollywood et al., 2007) y muchos empleadores tienen una actitud negativa hacia los trabajadores mayores (Fuertes et al., 2013).

La experiencia de varios países modernos muestra que en la tercera edad existen amplias oportunidades para preservar la actividad laboral de las personas involucrándolas en el emprendimiento, es decir, para crear sus propios negocios (Kautonen, 2008). El envejecimiento de la población es generalizado, por tanto, como lo demuestran una serie de estudios científicos (Kibler et al., 2012; Kraus \& Bouncken, 2014), en los próximos años, más personas de la tercera edad participarán en el emprendimiento, y utilizar una alternativa (en comparación con la mano de obra contratada) la posibilidad de ganar. En estos trabajos, también se sugirió que, debido al aumento de la esperanza de vida de las personas, habrá un aumento significativo en la participación de nuevas empresas creadas por empresarios pertenecientes a la tercera edad en el total de empresas de nueva creación.

Atraer a personas de la tercera edad para que creen sus propias empresas es un problema urgente en Rusia, donde el espíritu empresarial se ha desarrollado desde 1991. Actualmente, las pequeñas y medianas empresas están muy extendidas en Rusia. La mayoría de estas empresas son eficientes y rentables (Pinkovetskaia, 2019); operan en todas las regiones de Rusia y en la gran mayoría de las actividades económicas (Kiseleva et al., 2019). En relación con el aumento de la edad de jubilación, se puede suponer que en los próximos años aumentará considerablemente el número de personas mayores de 55 años en Rusia. 


\section{Revisión de literatura}

Las características de los primeros emprendedores de la tercera edad, como se señala en la literatura, incluyen beneficios sociales y económicos potenciales como la prolongación de la actividad activa de la población anciana debido al surgimiento de una opción de empleo alternativa, una disminución en el número de terceros. edad desempleada y la promoción de la actividad social de dichos ciudadanos (Kautonen et al., 2013). Los estudios (Abrosimova, 2011; Botham \& Graves, 2009) sugieren que los emprendedores de edad avanzada pueden asegurar el crecimiento de la innovación en la producción mediante el uso de sus competencias y conocimientos. Un estudio (Zhang, 2014) mostró ventajas de las actividades de los emprendedores como una mayor flexibilidad en los horarios de producción, falta de control constante y libertad para planificar sus actividades. Estos factores son especialmente importantes para aquellas personas que dedican mucho tiempo a familiares ancianos o enfermos, así como a nietos. Para las personas que planean jubilarse, el emprendimiento a pequeña escala es una opción interesante para continuar con un estilo de vida activo y mantener su inclusión social (Thorgren et al., 2016). Una de las características esenciales del espíritu empresarial temprano en la tercera edad es la recepción de los denominados dividendos no monetarios. Reflejan los aspectos sociales y los intereses personales de los empresarios mayores, como el deseo de autonomía, la autorrealización y el deseo de las personas de sentirse activas, útiles y valiosas para sus familiares (Soto-Simeone \& Kautonen, 2020; Baron et al. , 2016). También se observa en Rusia un aumento de la esperanza de vida de las personas, como tendencia al envejecimiento de la población. Este fenómeno determina la necesidad de la formación de una nueva política de Estado en el ámbito del empleo de la población en edad de prejubilación y jubilación. La situación se ve agravada por las consecuencias socioeconómicas de los cambios en la legislación de pensiones rusa, que elevó la edad correspondiente, y en particular, el aumento esperado del desempleo, como se indica en el artículo (Kashepov, 2019). Es necesario entender que año tras año la población envejecerá y se incrementarán las necesidades de recursos laborales de la economía nacional, incluidas las personas pertenecientes a la tercera edad, teniendo en cuenta sus competencias, conocimientos y experiencia. Alentarlos a continuar con su vigorosa actividad se ha convertido en una de las principales orientaciones de la correspondiente Estrategia aprobada por el Gobierno de Rusia (Estrategia para acciones, 2016). La estrategia prevé medidas para garantizar la recepción de fondos adicionales por parte de los ciudadanos de las generaciones mayores, la aplicación de sus conocimientos y habilidades en los procesos económicos, así como para mejorar el estatus social de estos ciudadanos. En comparación con los colegas más jóvenes, los emprendedores de la tercera edad tienen una serie de características específicas, enfrentan diferentes barreras y necesitan un apoyo diferente (Gielnik et al., 2018). En base a esto, la formación de las medidas de asistencia y apoyo que necesitan los empresarios mayores debe basarse en la comprensión del fenómeno de la actividad emprendedora en la tercera edad. Esto determina la relevancia de analizar los patrones inherentes al emprendimiento temprano en la tercera edad en Rusia, a lo que está dedicado este artículo. El artículo examina las ventajas y desventajas propias de los primeros emprendedores en la tercera edad, y la valoración del nivel actual de dicho emprendimiento en nuestro país. Los términos emprendedores tempranos $\mathrm{y}$ emprendimiento temprano se refieren a las actividades de personas que han estado involucradas en la creación de sus propios negocios o que los han tenido durante menos de tres años y medio. Los investigadores consideraron ciertos problemas del espíritu empresarial de la tercera edad en Rusia utilizando el ejemplo de regiones individuales de nuestro país. Entonces, en el artículo (Potekhina \& Chizhov, 2016) se muestra en el ejemplo de las regiones del Distrito Federal Central de Rusia la falta de oportunidades de empleo para las personas mayores. Por tanto, para la parte activa de estas personas, la única forma de mantener actividades interesantes pasa a ser emprendimiento temprano. La situación se complica por el hecho de que un aumento de la edad de jubilación en Rusia en 2019 supondrá un aumento del número de personas que aún no perciben pensiones, que, por las peculiaridades de la legislación vigente, tendrán dificultades para encontrar un trabajo. Como se desprende del trabajo (Smirnova, 2019), se puede suponer que estas personas centrarán su atención en la creación de sus propios negocios. El artículo (Popova \& Zorina, 2016) presenta los resultados de una encuesta sociológica sobre los problemas de la tercera edad en los municipios de la República de Komi, que mostró que solo el $1 \%$ de la población tiene su propio negocio. Un nivel significativamente más alto de emprendimiento en la tercera edad (2.8\%) fue mostrado por una encuesta de cuestionario de la población de la República de Karelia (Karginova-Gubinova et 
al., 2018). Al mismo tiempo, se ha prestado poca atención al problema de la actividad empresarial temprana de las personas de la tercera edad en Rusia en la investigación científica nacional.

\section{Metodología}

Hay tres aspectos que determinan la relevancia del problema del emprendimiento de la tercera edad en Rusia. La tendencia general de envejecimiento de la población señalada anteriormente y una disminución en el número de personas jóvenes y maduras conducirá a un aumento en la necesidad de la economía del país de atraer a las generaciones mayores al trabajo. La tendencia al envejecimiento de la población está confirmada por las estadísticas oficiales, que muestran que persiste durante muchos años. Entonces, en comparación con 1979, el número de personas cuya edad estaba en el rango de 55 a 69 años en 2018 aumentó en un 78\%, mientras que la población total en Rusia aumentó solo en un $7 \%$. El segundo aspecto está asociado a las dificultades existentes en el empleo de la población en edad de prejubilación y jubilación. La situación se ha agravado en la actualidad, debido al aumento de la edad de jubilación establecida legislativamente de acuerdo con la reforma, en la que los empresarios temen aceptar trabajadores en edad de prejubilación. El tercer aspecto viene determinado por el deseo de los pensionistas de ampliar su actividad laboral. Los motivos de esto pueden ser tanto la necesidad de aumentar los ingresos disponibles como de mantener la actividad social, siempre que la salud lo permita. Esto se describe en detalle en el artículo (Kolesnikova, 2019), que indica que en Rusia la continuación de la actividad laboral por parte de personas de la tercera edad tiene un efecto positivo en el estado psicológico de las personas, aumenta su estatus social y prestigio en la familia. . Este artículo también concluye que el nivel educativo de los ciudadanos pertenecientes a la tercera edad está cambiando. La proporción de personas con educación superior está creciendo, lo cual es importante, ya que son estas personas las que prevalecen entre los potenciales emprendedores.

El propósito de nuestro estudio fue identificar las características de las actividades de los emprendedores en la tercera edad en Rusia y la dinámica de este emprendimiento en los últimos años y en el futuro. El estudio incluyó tres etapas. En la primera etapa, analizamos la dinámica del número de emprendedores en etapa inicial de la tercera edad para el período de 2013 a 2018 en Rusia. En la segunda etapa, se llevó a cabo un análisis comparativo de la actividad empresarial en Rusia y varios países grandes económicamente desarrollados. En la tercera etapa, se realizó una evaluación del número potencial de nuevos emprendedores en Rusia para el futuro hasta 2035. Se tomó en cuenta que las personas mayores de 65 años relativamente rara vez inician nuevos negocios (Bates, 1995). Por tanto, en nuestro estudio se evaluaron los indicadores de emprendimiento temprano para el grupo de edad (55-64 años). Como datos iniciales para el estudio, se utilizaron los resultados de las encuestas realizadas en el proceso del (Global Entrepreneurship Monitor, 2019), el proyecto correspondiente en Rusia (National report, 2017), así como información estadística oficial sobre la estructura de edad del población y el pronóstico demográfico para Rusia (Servicio de estadísticas del estado federal, 2020). Con base en esto, consideramos tres opciones de pronóstico: bajo, medio y alto.

\section{Resultados científicos y discusión}

En la primera etapa del trabajo, analizamos la dinámica del número de emprendedores de la tercera edad en Rusia en los últimos años. La Tabla 1 muestra datos sobre la población de 55 a 64 años en Rusia, la proporción de emprendedores en etapa inicial de esta edad y el número de emprendedores. Los cálculos correspondientes se realizaron sobre la base de la información proporcionada en el Informe de Seguimiento del Emprendimiento, así como las estadísticas oficiales.

Tabla 1.

Características de la población y emprendedores en la tercera edad.

\begin{tabular}{llll}
\hline Años & $\begin{array}{l}\text { Población, miles de } \\
\text { personas }\end{array}$ & $\begin{array}{l}\text { Proporción de } \\
\text { emprendedores de la } \\
\text { tercera edad }\end{array}$ & $\begin{array}{l}\text { Monto } \\
\text { los primeros en adoptar, } \\
\text { miles personas }\end{array}$ \\
\hline 1 & 2 & 3 & 4 \\
2013 & 19072 & 0.099 & 1888 \\
2014 & 19583 & 0,076 & 1488 \\
2016 & 20538 & 0,043 & 883 \\
2018 & 20832 & 0,020 & 437 \\
\hline
\end{tabular}

Fuente: Elaboración propia a partir de datos estadísticos. 
Los datos proporcionados en la columna 2 del Cuadro 1 muestran que la población de 55 a 64 años aumentó significativamente (en 1,7 millones de personas) durante el período de 2013 a 2018. Un análisis de la información proporcionada en la tercera columna muestra una disminución constante en la proporción de emprendedores en etapa inicial en la población total de Rusia. A pesar del crecimiento de la población, el número total de emprendedores de nueva creación en los últimos años ha disminuido significativamente (en 4,3), como puede verse en los datos que figuran en la cuarta columna de la tabla. Consideremos las principales razones de este fenómeno. La principal razón del fuerte descenso de la actividad empresarial temprana en la tercera edad es, en nuestra opinión, la crisis de la economía rusa que comenzó en 2015. La crisis se asoció con una disminución de la actividad económica, una disminución de la producción y el consumo y la inflación. Esto condujo a una disminución de los ingresos reales de la población, una caída de la demanda y las restricciones sobre la financiación y la inversión (Bulletin of the socio-economic crisis in Russia, 2015). En consecuencia, disminuyó la demanda de la población por bienes y servicios del sector empresarial, disminuyó el trabajo que realizan los empresarios para las grandes corporaciones y empresas, así como para las organizaciones estatales y municipales. Todo esto tuvo el mayor impacto en los aspirantes a emprendedores de la tercera edad debido a su limitada competitividad. Además, debe tenerse en cuenta que las estructuras financieras y crediticias, de arrendamiento y otras estructuras desconfían de los empresarios principiantes de esta época. Junto con la crisis, la disminución del número de emprendedores de la tercera edad se debe al aumento de los requisitos para las empresas por parte de los organismos gubernamentales (fiscal, fiscalizador, administrativo). La necesidad de redactar un gran número de documentos que no están directamente relacionados con las competencias profesionales de los emprendedores, muchos de los cuales son bastante difíciles de redactar, complica significativamente la actividad. El uso de especialistas externos es bastante caro. Dominar todos los conocimientos necesarios, dado el corto plazo de la actividad propuesta (horizonte de planificación), parece irracional para muchos empresarios potenciales. Teniendo esto en cuenta, una parte de los posibles emprendedores de nueva creación detiene sus intentos de crear su propio negocio, mientras que la otra parte prefiere realizar sus actividades sin un registro oficial. Como se indica en el estudio (Burov, 2019), un análisis del sector empresarial en Rusia mostró que, en las condiciones económicas, legales e institucionales existentes, las actividades de los emprendedores en los últimos años se han vuelto ineficaces. Por tanto, muchos emprendedores, para reducir este impacto negativo, trasladan sus actividades al sector sombra de la economía. Al mismo tiempo, en ciertos tipos de actividades, como trabajos de reparación y construcción, transporte, servicios personales y sociales, alquiler, la economía sumergida alcanza hasta el $50 \%$ de la producción en Rusia.

Tabla 2.

Actividad emprendedora temprana en la tercera edad en las grandes economías.

\begin{tabular}{llll}
\hline El país & $\begin{array}{l}\text { Proporción de } \\
\text { emprendedores de la tercera } \\
\text { edad }\end{array}$ & El país & $\begin{array}{l}\text { Proporción de } \\
\text { emprendedores de la tercera } \\
\text { edad }\end{array}$ \\
\hline Italia & 0,021 & Gran Bretaña & 0,062 \\
Alemania & 0,024 & $\begin{array}{l}\text { India } \\
\text { Canadá }\end{array}$ & 0,069 \\
Japón & 0,026 & Brasil & 0.093 \\
Francia & 0,040 & EE.UU & 0,097 \\
España & 0,047 & $\begin{array}{l}\text { Promedio de los países } \\
\text { analizados }\end{array}$ & 0,104 \\
China & 0,051 & 0,058 \\
\hline
\end{tabular}

Fuente: Calculado por los autores con base en Global Entrepreneurship Monitor ( 2019 )

La tabla anterior muestra la información de que la proporción de emprendedores de la tercera edad de los países en cuestión está en el rango de 0.021 a 0,104 . Es decir, existe una diferenciación significativa de valores de estos parámetros. En los países en desarrollo (China, India, Brasil), los valores de los indicadores son más altos que en la mayoría de los países económicamente desarrollados. El alto nivel de emprendedores en etapa temprana de la tercera edad en los Estados Unidos y Canadá debido a la corriente en los últimos años en estos países, la tendencia hacia el desarrollo del autoempleo, incluso para ingresos adicionales a la edad de prejubilación y jubilación. 
La comparación de los datos que se muestran en la Tabla 2 con los valores de la actividad empresarial temprana en Rusia muestra que en 2013 y 2014 los indicadores rusos superaron el valor promedio de los países considerados. En 2018, el nivel de actividad empresarial en Rusia se volvió más bajo que en todos los países extranjeros en consideración, lo que indica la presencia de problemas importantes en el desarrollo del espíritu empresarial de la tercera edad en Rusia.

En la tercera etapa del trabajo, y a partir del pronóstico desarrollado por el Servicio Federal de Estadísticas Estatales, proyección demográfica (Federal state statistics service,
2020), se evaluó el número potencial de nuevas empresas en el futuro hasta 2035. Los resultados de esta evaluación se muestran en la Tabla 3. Se presentan tres variantes de predicción (baja, media y alta) de acuerdo con tres de pronostico demográfico. La proporción de empresarios que pertenecen a la tercera edad, en el total de población de la correspondiente categoría de edad en la variante baja de las previsiones adoptadas como al nivel de 2018 en Rusia. De acuerdo con la versión promedio del pronóstico, este indicador se toma al nivel del valor promedio para países extranjeros (Cuadro 3). La proporción de emprendedores en fase temprana de alta variante de la previsión adoptada como al nivel de 2013 en Rusia.

Tabla 3.

Características de la población y empresarios potenciales.

\begin{tabular}{llll}
\hline Años & $\begin{array}{l}\text { Población, millones } \\
\text { de personas }\end{array}$ & $\begin{array}{l}\text { Proporción de } \\
\text { emprendedores de la tercera } \\
\text { edad }\end{array}$ & $\begin{array}{l}\text { Número potencial } \\
\text { empresarios de la tercera edad, } \\
\text { millones de personas }\end{array}$ \\
\hline uno & $\begin{array}{c}\text { dos } \\
\text { Opción de pronóstico bajo }\end{array}$ & tres & cuatro \\
2025 & 22,4 & 0,020 & 449 \\
2030 & 22,9 & 0,020 & 459 \\
2035 & 23,5 & 0,020 & 471 \\
Previsión media & & & \\
2025 & 22,6 & 0,058 & 1309 \\
2030 & 23,2 & 0,058 & 1348 \\
2035 & 24.0 & 0,058 & 1391 \\
Opción de pronóstico alto & & \\
2025 & 23,0 & 0.099 & 2279 \\
2030 & 24,4 & 0.099 & 2415 \\
2035 & 26,2 & 0.099 & 2591 \\
\hline
\end{tabular}

Fuente: Calculado por los autores.

Los datos de la Tabla 3 sugieren un número significativo de posibles usuarios tempranos en el futuro. Dada la tendencia al envejecimiento de la población y la reducción de su fuerza laboral, sin estos negocios fracasarán en el futuro, generando empleo en la edad de prejubilación y jubilación, reponiendo el presupuesto estatal y aumentando los ingresos reales. Por lo tanto, en nuestra opinión, es necesario, al desarrollar programas para el desarrollo de la economía rusa a mediano y largo plazo, asegurar el desarrollo del espíritu empresarial en la tercera edad, no inferior a la versión promedio de la previsión dada en la Tabla 3.

\section{Conclusiones}

Los resultados del estudio, que tienen novedad científica y originalidad, incluyen: un análisis de la dinámica del número de emprendedores tempranos de la tercera edad en Rusia para el período de 2013 a 2018, un análisis comparativo de la actividad empresarial en Rusia y un número de los grandes países económicamente desarrollados, así como una evaluación del número potencial de nuevos empresarios en Rusia. Rusia para el futuro hasta 2035.

Superar los problemas en el desarrollo del emprendimiento en la tercera edad que tuvo lugar en 2018 en Rusia e implementar los planes trazados por el Gobierno del país (Strategy for actions, 2016; Strategy for the development of small and medium entrepreneurship, 2016), parece oportuno desarrollar e implementar un conjunto de medidas para brindar asistencia y apoyo estatal a los emprendedores principiantes de la tercera edad, teniendo en cuenta las características de dichos emprendedores. El complejo especificado debe incluir las siguientes actividades. Apoyo financiero a los emprendedores, incluida la asignación de 
subsidios y subvenciones, la exención del impuesto sobre la renta después del inicio de la edad de jubilación y la disminución del nivel de los pagos sociales, y la provisión de garantías de préstamos. Apoyo a la propiedad, es decir, la provisión de locales y otros activos fijos en alquiler preferencial. Soporte de información y consultoría basado en el desarrollo de sistemas relevantes que operan tanto en línea como en departamentos gubernamentales especializados en ayudar a los emprendedores y brindarles información actualizada, así como brindarles asistencia en forma de asesoría profesional. Apoyo educativo, que incluye el desarrollo de programas especiales y formación gratuita para emprendedores de la tercera edad, teniendo en cuenta sus características. Estimular la salida de la economía sumergida de los emprendedores en edad de prejubilación y jubilación, especialmente los autónomos, a partir de proporcionarles diversos beneficios fiscales en función de la especificidad del tipo de actividad en la que se especializan. Desarrollo del emprendimiento intraempresarial, es decir, el traspaso de determinadas obras y servicios dentro de una empresa u organización a la subcontratación a cargo de especialistas de la tercera edad. Además, estas actividades se pueden realizar según sea necesario en un horario flexible.

Por supuesto, no todas las personas mayores pueden ser emprendedores de la tercera edad, ya que no todos tienen las habilidades e intenciones adecuadas y están preparados para crear sus propios negocios. Pero las personas de este grupo de edad, sin duda, tienen un potencial significativo que debe utilizarse correctamente. Fomentar el espíritu empresarial de la tercera edad puede ser una forma de abordar los problemas del envejecimiento de la población. Al convertirse en emprendedores, las personas de la tercera edad se mantienen activas, resuelven muchos de sus problemas, proporcionan un aumento del empleo y contribuyen al crecimiento económico. En general, como ha demostrado la investigación, se puede suponer que aumentará la participación de estos empresarios en los procesos económicos.

\section{Referencias}

Abrosimova, A. A. (2011). Attitude of entrepreneurs of different age groups to innovation activity. Economics and management, (12-2), 122-126.

Baron, R.A., Franklin, R.J., \& Hmieleski, K.M. (2016). Why entrepreneurs often experience low, not high, levels of stress: The joint effects of selection and psychological capital. Journal of Management, 42(3), 742-768.

Bates, T. (1995). Self-Employment Entry across Industry Groups. Journal of Business Venturing, 10, 143-156.

Botham, R., \& Graves A. (2009). Third age entrepreneurs: innovative business start-ups in mid-life and beyond-understanding the drivers and removing the barriers. Interim report to NESTA, February.

Bulletin of the socio-economic crisis in Russia. Russia's entry into the crisis (late 2014-early 2015). Analytical center under the government of the Russian Federation. April 2015. Recuperado de: http://ac.gov.ru/files/publication/a/5479.pdf (Accessed 19 October 2020).

Burov, V. I. (2019). Improving the system of state regulation of small business in the conditions of dominance of shadow economic activity. Shadow economy, (1), 9-16.

Federal state statistics service. Demography. Recuperado de: http://old.gks.ru/wps/wcm/connect/rosstat_main /rosstat/ru/statistics/population/demography/\# (Accessed 24 October 2020).

Fuertes, V., Egdell, V., \& McQuaid, R. (2013). Extending working lives: age management in SMEs. Employee Relations, 35 (3), 272-293.

Gielnik, M. M., Zacher, H., \& Wang, M. (2018). Age in the entrepre $\neg$ neurial process: the role of future time perspective and prior entrepreneurial experience. Journal Applied Psychologies, 103, 1067-1085

Gimmon, E., Yitshaki, R., \& Hantman, S. (2018). Entrepreneurship in the third age: retirees' motivation and intentions. International Journal Entrepreneurship and Small Business, 3, 267-288.

Global Entrepreneurship Monitor (2019). Global Entrepreneurship Research Association (GERA). Harasty, C., \& Ostermeier, M. (2020). Population ageing: Alternative measures of dependency and implications for the future of work. ILO Working Paper 5, June, 53 p.

Hollywood, E., Brown, R., Danson, M., \& McQuaid, R. (2007). Demographic and labour market change: the dynamics of older workers in the Scottish labour market. Scottish Geographical Journal, 123(4), 242-256.

Karginova-Gubinova, V. V., Romashkina, Yu. V., Prokopyev, E. A. (2018), Typology of labor behavior of third-age persons (on the example of the Republic of Karelia). Russian entrepreneurship, (10), 3231-3244.

Kashepov, A.V. (2019). Forecasting the socioeconomic consequences of changes in pension legislation for the period up to 2024. Bulletin of the Russian new University. Series " Man and society", (1), 57-66. 
Kautonen, T., Down, S., \& Minniti, M. (2013). Ageing and entrepreneurial preferences. Small Business Economics, 42(3), 579-594.

Kautonen, T. (2008). Understanding the older entrepreneur: Comparing third age and prime age entrepreneurs in Finland. International Journal of Business Science and Applied Management, 3(3), 3-13.

Kibler, E., Wainwright, T., Blackburn, R., \& Kautonen, T. (2012). Under pressure: differentiation in older entrepreneur motivations, emotions and mentoring. In 35th Institute for Small Business and Entrepreneurship (ISBE) Annual Conference, Dublin, IE.

Kiseleva, O., Lebedev, A., Pinkovetskaia, I., Rojas-Bahamón, M., \& Arbeláez Campillo, D. (2019). Specialization and concentration of small and medium enterprises employees: Russian data. Amazonia Investiga, 8(20), 6-15. Recuperado

de https://amazoniainvestiga.info/index.php/amazo nia/article/view/59

Kolesnikova, O. A. (2019), Elderly people in the labor market. Bulletin of the Voronezh Institute of high technologies, 1(28), 165-168.

Kraus, S., \& Bouncken, R. B. (2014). Senior entrepreneurship: definitions and underlying theories. International Journal of Business Research, 14(3), 209-215.

Loretto, W. (2010). Work and retirement in an ageing world: the case of older workers in the UK. Journal of the Academy of Social Sciences, 5(3), 279-294.

Maestas, N., \& Zissimopoulos, J. (2010). How Longer Working Lives Ease the Crunch of Population Ageing. The Journal of Economic Perspectives, 24(1). 139-160.

National report. (2017). Global entrepreneurship monitor. Russia 2016/2017. O. R. Verkhovskaya, E. A. Alexandrova, K. A. Bogatyreva, M. V. Dzhelepova, E. V. Shmeleva. Higher school of management of Saint Petersburg state University. Recuperado de smb.gov.ru/files/images/gem_russia_20162017.pdf (Accessed 24 November 2020).
Popova, L. A., \& Zorina, E. N. (2016). Standard of living and resource potential of pensioners in the Northern regions of Russia. Region: Economics and sociology, 1(89), 135-158. Potekhina, I. P., \& Chizhov, D. V. (2016). The potential of the older generation as a component of the national human capital (based on research in the regions of the CFD). Monitoring of public opinion. Economic and social changes, (2), 3-23. Smirnova, A.V. (2019). Involvement of older people in entrepreneurial activity in connection with the pension reform of the Russian Federation. Management of economic systems: electronic scientific journal, 1(119), 1-10.

Soto-Simeone, A., \& Kautonen, T. (2020). Senior entrepreneurship following unemployment: a social identity theory perspective. Review of Managerial Science. May, 1-40.

Strategy for actions in the interests of older citizens in the Russian Federation until 2025: decree Of the Government of the Russian Federation of 5 February 2016. № 164-r.

Strategy for the development of small and medium entrepreneurship in the Russian Federation for the period up to 2030: Government Decree No. 1083-R of June 2, 2016. Collection of legislation of the Russian Federation, 2016, $\mathrm{N}^{\circ} 24,3549$.

Thorgren, S., Sirén, C., Nordström, C., \& Wincent J. (2016). Hybrid entrepreneurs' second-step choise: The nonlinear relationship between age and intention to enter full-time entrepreneurship. Journal of Business Venturing Insights, 5, 14-18.

Zhang, T. (2014). Elderly Entrepreneurship in an Aging US Economy: It's Never Too Late, World Scientific Books, World Scientific Publishing Company. 284

World Population Ageing (2019). Highlights. Department of Economic and Social Affairs Population Division. United Nations. New York, 46. 